\title{
Fudge Factors in the Birch and Swinnerton-Dyer Conjecture
}

\author{
Karl Rubin
}

The aim of this note is to describe how the "fudge factors" in the Birch and Swinnerton-Dyer conjecture vary in a family of quadratic twists (see Proposition 5, which follows directly from Tate's algorithm $[\mathrm{T}])$. We illustrate with two examples.

Definition 1. If $E$ is an elliptic curve over $\mathbf{Q}$ and $p$ is a prime, the fudge factor (or Tamagawa factor) $c_{p}(E)$ is defined by

$$
c_{p}(E)=\left[E\left(\mathbf{Q}_{p}\right): E_{0}\left(\mathbf{Q}_{p}\right)\right]
$$

where $E_{0}\left(\mathbf{Q}_{p}\right)$ is the subgroup of $E\left(\mathbf{Q}_{p}\right)$ consisting of those points whose reduction modulo $p$ (on a minimal model of $E$ ) is nonsingular.

The fundamental method for computing the fudge factors is Tate's algorithm. This algorithm, originally described in a 1965 letter to Cassels, was published in $[\mathrm{T}]$ and essentially reproduced in $\S I V .9$ of $[\mathrm{S}]$. Standard number theoretic computer packages, such as PARI/GP (available at http://pari.math.u-bordeaux.fr), will compute these factors very efficiently.

Let $\Delta(E)$ denote the discriminant of a minimal model of $E$.

Proposition 2. Suppose $E$ is an elliptic curve over $\mathbf{Q}$.

1. If $E$ has good reduction at $p$, then $c_{p}(E)=1$.

2. If $E$ has split multiplicative reduction at $p$, then $c_{p}(E)=\operatorname{ord}_{p}(\Delta(E))$, i.e., $p^{c_{p}(E)}$ is the highest power of $p$ dividing $\Delta(E)$.

3. If $E$ has nonsplit multiplicative reduction at $p$, then $c_{p}(E) \leq 2$ and $c_{p}(E) \equiv \operatorname{ord}_{p}(\Delta(E))(\bmod 2)$.

4. If $E$ has additive reduction at $p$, then $c_{p}(E) \leq 4$.

Proof. These are cases 1, 2a, 2b, and 3 through 10, respectively, of Tate's algorithm $[\mathrm{T}]$.

\footnotetext{
${ }^{0}$ Supported by NSF grant DMS-0140378.
} 
Fix an elliptic curve $E$ and a model of $E$ of the form

$$
y^{2}=f(x)
$$

with a monic cubic polynomial $f(x) \in \mathbf{Z}[x]$, and let $\Delta$ denote the discriminant of this model. We may assume that the model is minimal at all primes $p>2$, but this is not necessary for what follows.

Definition 3. The quadratic twist of $E$ by a nonzero rational number $d$ is

$$
E_{d}: y^{2}=d^{3} f(x / d) .
$$

We will write simply $c_{p}(d)$ for $c_{p}\left(E_{d}\right)$. The purpose of this note is to describe how $c_{p}(d)$, and $\prod_{p} c_{p}(d)$, vary with $d$.

Lemma 4. Suppose $d, d^{\prime} \in \mathbf{Q}^{\times}$.

1. If $d / d^{\prime}$ is a square in $\mathbf{Q}$, then $E_{d}$ is isomorphic to $E_{d^{\prime}}$.

2. If $p$ is a prime and $d / d^{\prime}$ is a square in $\mathbf{Q}_{p}$, then $c_{p}(d)=c_{p}\left(d^{\prime}\right)$.

Proof. If $d^{\prime}=d r^{2}$, then the map $(x, y) \mapsto\left(r^{2} x, r^{3} y\right)$ is an isomorphism from $E_{d}$ to $E_{d^{\prime}}$. If $r \in \mathbf{Q}^{\times}$, this proves (i). If $r \in \mathbf{Q}_{p}^{\times}$, this isomorphism identifies $E_{d}\left(\mathbf{Q}_{p}\right)$ with $E_{d^{\prime}}\left(\mathbf{Q}_{p}\right)$ and by the definition of $c_{p}(d)$ we get $c_{p}(d)=c_{p}\left(d^{\prime}\right)$.

By Lemma 4(i), every quadratic twist $E_{d}$ of $E$ is a twist by some (unique) squarefree integer. From now on we will assume that $d$ is a squarefree integer. Proposition 5. Suppose $p$ is a prime not dividing $2 \Delta$. If $p \nmid d$ then $c_{p}(d)=1$. If $p \mid d$, then

$$
c_{p}(d)=1+\#\{\text { roots of } f(x) \equiv 0(\bmod p) \text { in } \mathbf{Z} / p \mathbf{Z}\}=1,2 \text {, or } 4 .
$$

Proof. If $p \nmid 2 \Delta d$ then $E_{d}$ has good reduction at $p$, so $c_{p}(d)=1$. If $p \mid d$ but $p \nmid 2 \Delta$ then we are in case 6 of Tate's algorithm [T].

Note that for every $p$ not dividing $2 \Delta$, the number of roots of $f(x)$ modulo $p$ is at least as large as the number of roots of $f(x)$ in $\mathbf{Q}$. Thus if $p \mid d$ and $p \nmid 2 \Delta$, then $c_{p}(d) \geq \# E(\mathbf{Q})[2]$.

If $p \mid 2 \Delta$ the situation is more complicated. However, for those primes, to determine $c_{p}(d)$ for every $d$, Lemma 4 (ii) shows that it is enough to compute $c_{p}(d)$ (using Tate's algorithm) for $d$ in a set of representatives of $\mathbf{Q}_{p}^{\times} /\left(\mathbf{Q}_{p}^{\times}\right)^{2}$. Note that $\mathbf{Q}_{p}^{\times} /\left(\mathbf{Q}_{p}^{\times}\right)^{2}$ has order 4 if $p>2$, and order 8 if $p=2$.

Example 6. E : $y^{2}=x^{3}-x$

We have $\Delta=64$, and $x^{3}-x$ factors into linear factors over $\mathrm{Q}$, so Proposition 5 shows that for $p>2$ we have

$$
c_{p}(d)= \begin{cases}1 & \text { if } p \nmid d, \\ 4 & \text { if } p \mid d .\end{cases}
$$


Tate's algorithm (cases 4 and 7.2, respectively) gives

$$
c_{2}(d)= \begin{cases}2 & \text { if } 2 \nmid d, \\ 4 & \text { if } 2 \mid d .\end{cases}
$$

(Alternatively, we can use PARI/GP to compute that

$$
\begin{aligned}
& c_{2}(1)=c_{2}(3)=c_{2}(-1)=c_{2}(-3)=2, \\
& c_{2}(2)=c_{2}(6)=c_{2}(-2)=c_{2}(-6)=4,
\end{aligned}
$$

and then use Lemma 4(ii) to deduce (2).)

Combining (1) and (2) we conclude that

$$
\prod_{p} c_{p}(d)= \begin{cases}2^{2 \omega(d)+1} & \text { if } d \text { is odd } \\ 2^{2 \omega(d)} & \text { if } d \text { is even }\end{cases}
$$

where $\omega(d)$ is the number of prime divisors of $d$.

Example 7. E: $y^{2}+y=x^{3}-x^{2}-10 x-20$

This is the modular curve $X_{0}(11)$, with discriminant $-11^{5}$. We will use the model (not minimal at 2)

$$
y^{2}=x^{3}-4 x^{2}-160 x-1264
$$

with discriminant $\Delta=-2^{12} 11^{5}$. For $p \neq 2,11$, Proposition 5 shows that

$$
c_{p}(d)= \begin{cases}1 & \text { if } p \nmid d, \\ 1+\#\left\{\operatorname{roots} \text { of } x^{3}-4 x^{2}-160 x-1264 \bmod p\right\} & \text { if } p \mid d .\end{cases}
$$

Since $x^{3}-4 x^{2}-160 x-1264$ is irreducible over $\mathbf{Q}, c_{p}(d)$ can be 1,2 , or 4 . More precisely, the Galois group of $x^{3}-4 x^{2}-160 x-1264$ over $\mathbf{Q}$ is $S_{3}$, so the Cebotarev theorem shows that if $D_{k}$ is the density of the set of primes $p$ such that $x^{3}-4 x^{2}-160 x-1264$ has $k$ roots modulo $p$, then $D_{0}=1 / 3, D_{1}=1 / 2$, and $D_{3}=1 / 6$.

We also compute

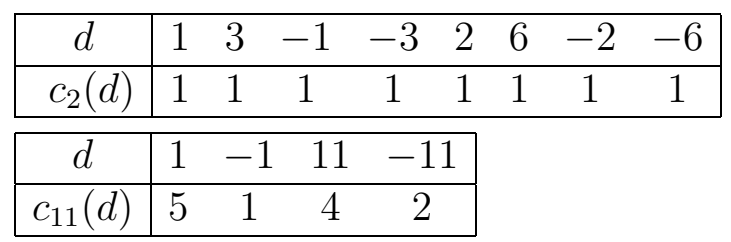

Therefore by Lemma $4(\mathrm{ii}), c_{2}(d)=1$ for every $d$, and

$$
c_{11}(d)= \begin{cases}5 & \text { if } d \text { is a nonzero square modulo } 11 \\ 1 & \text { if } d \text { is not a square modulo } 11 \\ 4 & \text { if } 11 \mid d \text { and } \frac{d}{11} \text { is a square modulo } 11 \\ 2 & \text { if } 11 \mid d \text { and } \frac{d}{11} \text { is not a square modulo } 11\end{cases}
$$




\section{References}

[S] J. H. Silverman, Advanced Topics in the Arithmetic of Elliptic Curves, Graduate Texts in Mathematics 151, New York: Springer-Verlag (1994).

$[\mathrm{T}] \quad$ J. Tate, Algorithm for determining the type of a singular fiber in an elliptic pencil. In: Modular functions of one variable (IV), Lecture Notes in Math. 476, New York: Springer-Verlag (1975) 33-52.

Department of Mathematics,

Stanford University,

Stanford, CA

94305 USA 